\title{
Von Onlineplattformen und mittelalterlichen Märkten
}

\author{
Gleichgewichtsmodelle und agentenbasierte Modellierung \\ zweiseitiger Märkte
}

\author{
Claudius Gräbner, Institut für die Gesamtanalyse der Wirtschaft (ICAE), Johannes Kepler Universität Linz, \\ Altenbergerstraße 52, 4040 Linz (claudius.graebner@jku.at), (1) orcid.org/0000-0003-4065-4722 \\ Torsten Heinrich, Institute for New Economic Thinking (INET) at the Oxford Martin School, University of Oxford \\ (torsten.heinrich@maths.ox.ac.uk), (1) orcid.org/0000-0001-8873-0806
}

Mit der zunehmenden Digitalisierung und Vernetzung ökonomischer Systeme haben plattformbasierte Interaktionsbeziehungen stark an Bedeutung gewonnen. Hier werden zwei oder mehr Nutzungsgruppen durch eine dritte Seite, die Plattform, zusammengebracht. Die Interaktion wird in solchen zweiseitigen Märkten von den Plattformbetreibern kontrolliert. Kreditkartensysteme, Softwaremärkte oder werbefinanzierte Online-Communities stellen typische Beispiele dar. Da die Analyse von zweiseitigen Märkten mit Gleichgewichtsmodellen aufgrund der algebraischen Struktur des Problems in der Regel nicht praktikabel ist, präsentieren wir im vorliegenden Beitrag ein agentenbasiertes Modell, welches in einem kürzlich erschienenen Artikel ausführlicher diskutiert wird. Anhand von illustrativen Beispielen verdeutlichen wir die Implikationen eines agentenbasierten Ansatzes für die Innovationsökonomik im Allgemeinen und das Studium von Technologieentwicklung im Besonderen.

\section{Of Online Platforms and Medieval Markets}

Equilibrium Models and Agent-Based Modeling of Two-Sided Markets

Platform-based interactions are much more important in today's increasingly digitalized and interconnected economic systems than they used to be in traditional ones. These interactions refer to markets where two or more user groups interact with the help of a third party, the platform. In such two-sided markets, the interaction is controlled by the platform provider. Common examples include credit card systems, software markets, and advertisement-financed online communities. As the analysis of two-sided markets largely defies modeling with equilibrium models due to the algebraic structure of the problem, the present contribution proposes an agent-based model as an alternative. It refers to a recently published article that discusses the agent-based model in

This is an article distributed under the terms of the Creative Commons Attribution License CCBY 4.0 (https://creativecommons.org/licenses/by/4.0/)

https://doi.org/10.14512/tatup.26.3.23

Submitted: 05. 07.2017. Peer reviewed. Accepted: 27.09.2017 more detail. Several examples illustrate the implications of the agentbased modeling approach for innovation economics and the study of technological development in particular.

KEYWORDS: two-sided markets, network externalities, agent-based computational economics, heuristic decision making, reinforcement learning

\section{Einleitung}

Das Thema dieses Beitrags sind zweiseitige Märkte. Wir sprechen von einem zweiseitigen Markt, wenn Anbieter ${ }^{1}$ und potenzielle Kunden eines Produkts oder einer Dienstleistung nur über eine dritte Partei, Netzwerkbetreiber, Transaktionen miteinander durchführen können. Dies unterscheidet sie von herkömmlichen Märkten, in denen Käufer und Verkäufer direkt miteinander interagieren können.

Beispiele für zweiseitige Märkte sind Börsen und Märkte für Kreditkarten, Videospiele oder PCs. Die Netzwerkbetreiber spielen immer die gleiche Rolle, treten jedoch in unterschiedlicher Form auf: Im Falle von PCs sind die Anbieter Hersteller von Gebrauchssoftware, die Kunden Nachfrager dieser Software und der Netzwerkbetreiber der Anbieter eines Betriebssystems, auf dem die Gebrauchssoftware installiert werden kann. Eine Transaktion macht für die Anbieter und Nachfrager der Gebrauchssoftware nur Sinn, wenn beide über ein entsprechendes Betriebssystem verfügen: Die Anbieter müssen ggf. eine Lizenz erwerben und die Kunden müssen das Betriebssystem auf ihren PCs installieren. Auch wenn der Handel nicht direkt über den PC abgewickelt wird, stellt das Betriebssystem hier die „Platt-

1 Im Falle abstrakter Entitäten, die etwa Käuferseite und Verkäuferseite im Modell bezeichnen, verzichten wir auf gegenderte Formen. 
form“ dar, die beide Seiten des Marktes miteinander verbindet. Die Begriffe der Plattform und des Netzwerkbetreibers sind also breit zu verstehen: Es handelt sich nicht zwangsläufig um eine Handelsplattform, sondern um ein Produkt, das Anbieter und Nachfrager miteinander verbindet. In manchen Fällen, wie Börsenplattformen (z. B. Xetra), ist die Rolle der Netzwerkbetreiber aber tatsächlich die einer Handelsplattform.

Die Funktion zweiseitiger Märkte unterscheidet sich von der Funktion herkömmlicher Märkte. Ein Grund sind positive Feedbackschleifen: Ein Netzwerkbetreiber wird für Anbieter und Nachfrager attraktiver, je mehr Kunden des anderen Typs den Service des Netzwerkbetreibers in Anspruch nehmen. Je mehr Käufer bei einem Netzwerk registriert sind, desto größer ist der potenzielle Absatzmarkt für Verkäufer, und je mehr Verkäufer bei einem Netzwerk registriert sind, desto größer ist das Angebot für die Käufer. Diese Effekte werden Netzwerkeffekte genannt und können dafür sorgen, dass der Markt sich nicht zu einem stabilen Gleichgewicht zwischen Angebot und Nachfrage entwickelt, sondern von dauerhaften Dynamiken gekennzeichnet wird. Dies stellt eine Herausforderung für die in der Ökonomik weit verbreiteten Gleichgewichtsmodelle dar. Ökonom*innen sprechen von einem Marktgleichgewicht, wenn das Angebot für ein Gut der Nachfrage entspricht, sodass Angebotsmenge und Preis stabil bleiben. Diese Modelle sind nur dann anwendbar, wenn das Marktumfeld und die Entscheidungsprobleme der beteiligten Akteure derartig formalisiert werden können, dass ein solches Gleichgewicht existiert - und im besten Falle auch stabil ist. Dies ist bei zweiseitigen Märkten aufgrund des selbstreferenziellen Entscheidungsproblems von Kunden und Anbietern nicht sierten Modellen empfehlen wir den Beitrag von Hoffmann und Roos (2012).

Um die Vorzüge eines agentenbasierten Ansatzes zu illustrieren, gehen wir folgendermaßen vor: Im nächsten Abschnitt werden wir anhand von Beispielen die empirische Relevanz zweiseitiger Märkte verdeutlichen. Anschließend werden wir herkömmliche Ansätze zur Untersuchung zweiseitiger Märkte und ihre Probleme beschreiben. Im vierten Abschnitt führen wir ein agentenbasiertes Modell von zweiseitigen Märken ein, dessen Ergebnisse wir im fünften Abschnitt diskutieren. Der sechste Abschnitt fasst die zentralen Ergebnisse zusammen.

\section{Empirische Relevanz}

Plattformen in zweiseitigen Märkten müssen Unsicherheiten in wirtschaftlichen Austauschbeziehungen reduzieren. Wir wollen dazu das Beispiel von Onlinemarktplätzen betrachten: Für Onlinehändler ist es wichtig, eine breite Kundenbasis zu erreichen und ihre Sichtbarkeit im Internet sicherzustellen. Auf der anderen Seite wollen die Kunden zwielichtige Anbieter vermeiden. Sie bevorzugen daher ebenfalls weitläufig genutzte Plattformen. Die Plattform als dritter Akteur hat einen Anreiz, gegen Betrug, asymmetrische Information und Marktmacht vorzugehen, um ihre eigene Marktmacht auszubauen und auszunutzen. Die bekanntesten Beispiele für zweiseitigen Onlinemarktplätze sind eBay, Uber, Amazon, Alibaba oder Airbnb (z. B. Eisenmann et al. 2011). Verlässliche Daten über die Größe dieser Onlinemarktplätze sind nicht offen verfügbar. Für Ebay, Amazon, und

\section{Plattformen in zweiseitigen Märkten müssen Unsicherheiten in wirtschaftlichen Austauschbeziehungen reduzieren.}

notwendigerweise der Fall. Zudem ist der Strategieraum für die Netzwerkbetreiber enorm groß: Sie können Transaktionspreise und Zugangspreise für Anbieter und Kunden jeweils getrennt voneinander setzen. Preise können dabei auch negativ sein, d.h. Netzwerkbetreiber können die Anbieterseite von der Kundenseite subventionieren lassen oder umgekehrt. In der Konsequenz ist es nicht mehr möglich, das Preissetzungsproblem des Netzwerkbetreibers in ein einfaches Optimierungsproblem zu überführen und ein eindeutiges Gleichgewicht sicherzustellen.

Hier stellen wir eine alternative Methode zur Analyse zweiseitiger Märkten vor: agentenbasierte Modelle (ABM). In ABM werden die beteiligten Akteure als digitale Agenten programmiert und ihre Interaktion ohne eine a priori Gleichgewichtsannahme simuliert. Dies erlaubt den Forscher*innen die Verwendung realistischerer Annahmen. Solche Modelle sind gut geeignet, die essenziellen Eigenschaften zweiseitiger Märkte abzubilden und die Funktion dieser Systeme besser zu verstehen. Für eine allgemeinverständliche Einführung zu agentenba-
Alibaba liegen Schätzungen von Statista (statista.com) und DMR (expandedramblings.com) jeweils zwischen 100 und 600 Millionen Nutzer, für Uber etwas darunter. Ihrer sozialen und ökonomischen Relevanz tut dies jedoch keinen Abbruch. Der Mechanismus spielt auch in unregulierten Teilen des Netzes eine wichtige Rolle, beispielsweise im Fall von Silk Road, Alphabay und ähnlichen Plattformen. Silk Road und Alphabay waren anonyme Onlinemarktportale, in denen v. a. illegale Waren gehandelt wurden. Die Größenordnung wurde durch das FBI mit über 200.000 Nutzern angegeben. Zentral für den Erfolg der Plattformen ist es, eine große Kundenbasis (auf beiden Seiten) aufzubauen, auch wenn hierfür zunächst Verluste hinzunehmen sind.

Die Rolle einer zentralisierten und technologisch gestützten Plattform ist erst mit dem Aufstieg der Informations- und Kommunikationstechnologie entstanden. Netzwerkbetreiber haben nun nicht nur alle Möglichkeiten der Preissetzung, sie kontrollieren auch die technischen Eigenschaften der Plattform und damit den Informationsfluss und die Restunsicherheit der An- 
bieter und Kunden. Sie wären in der Lage, die Plattform vorübergehend außer Betrieb zu nehmen und die Kompatibilität zu Konkurrenzplattformen zu vereinfachen oder zu erschweren.

Zweiseitige Märkte sind nur bedingt ein neues Phänomen. Da traditionelle ökonomische Handelsnetzwerke ebenfalls von Unsicherheit betroffen waren, besteht die Rolle der Plattform schon seit langer Zeit. Im spätmittelalterlichen und frühneuzeitlichen Mitteleuropa wurde sie - ganz bildlich - von Märkten in Städten wahrgenommen. Reisende Handelsleute kauften lokal produzierte Waren auf und importierten im Gegenzug andere Waren; sie mussten hierzu mit lokalen Anbieter*innen und Abnehmer*innen in Kontakt treten. Der Handel war hochgradig organisiert; ortsfremden Handelsleuten standen lokale Broker zur Verfügung, welche die Verbindung zu lokalen Produzenten oder Abnehmern herstellten. Das Geschäft der Broker war dabei durch die Städte strikt reguliert. Die Städte waren sehr darauf bedacht, den Handel, je nach Warengruppe, anbieter- oder abnehmerfreundlich zu organisieren. Die historische Entwicklung der Plattformen als Marktinstrument wird u. a. durch Boerner und Quint (2010) aufgearbeitet.

\section{Bestehende Theorien zweiseitiger Märkte}

Viele Modelle zweiseitiger Märkte sind spieltheoretischer Natur. Nachdem für Anbieter und Käufer eine Präferenzstruktur spezifiziert wurde, welche die Herleitung eines Gleichgewichts erlaubt, wird eine optimale Preispolitik der Plattform berechnet. Hierzu sind sehr stilisierte Annahmen notwendig, da die Anzahl der Anbieter mit Plattformzugang über die Netzwerkexternalität in die Nutzenfunktion der Nachfrageseite eingeht und umgekehrt. Eines der einflussreichsten Gleichgewichtsmodelle (Rochet und Tirole 2006) ist beispielhaft in Heinrich und Gräbner (2017) diskutiert.

Die Annahmen von Gleichgewichtsmodellen sind nicht nur für sich genommen unrealistisch, sie erlauben es auch nicht, die direkte Interdependenz von Anbietern und Nachfragern und die daraus resultierenden Netzwerkeffekte im Modell zu berücksichtigen. Zudem ist es oft notwendig anzunehmen, dass die Plattformbetreiber die Nachfragefunktion der Kunden kennen.

Neben Gleichgewichtsmodellen wurden eine Reihe empirischer Fallstudien (Börner und Quint 2010; Eisenmann et al. 2011) publiziert, die theoretisch jedoch oft in der Logik der Gleichgewichtsmodelle verankert sind. Sie tendieren dazu, den Akteuren Optimierungsentscheidungen und bewusste, perfekt informierte Manipulation von Gleichgewichten zu unterstellen. Es gibt vereinzelt evolutorische und agentenbasierte Modellierungsansätze (Peitz et al. 2011; Meyer 2012), die aber dennoch in Optimierungsframeworks arbeiten, und Modelle so strukturieren, dass die Optimierungsprobleme zumindest rechnerisch lösbar sind. Wir verwenden im Folgenden heuristische Entscheidungsmechanismen, deren Lösbarkeit weniger an bestimmte Modellstrukturen gebunden ist.

\section{Eine agentenbasierte Perspektive auf zweiseitige Märkte}

ABM erlauben es uns, die nicht-linearen Feedbackschleifen und Netzwerkeffekte, die charakteristisch für zweiseitige Märkte sind, direkt im Modell abzubilden. Zudem verzichten sie in der Regel auf eine a priori Gleichgewichtsannahme. Sie werden numerisch simuliert und können die Nicht-Gleichgewichtsdynamiken des Systems und seine Pfadabhängigkeit direkt abbilden. Dabei werden Nichtkonvergenz und Pfadabhängigkeit durch das weite Auseinandergehen der 10\% und 90\% Quantile in Abbildung 1 deutlich. Zwar werden auch herkömmliche Gleichgewichtsmodelle häufig numerisch gelöst, allerdings verfolgt die Simulation dann das Ziel, einen Gleichgewichtszustand zu identifizieren, aber nicht tatsächliche Prozesse zu imitieren, wie dies bei agentenbasierten Simulationen der Fall ist. Den interessierten Leser verweisen wir auf den wissenschaftsphilosophischen Beitrag von Lehtinen und Kuorikoski (2007), der diese Unterscheidung ausführlich diskutiert.

Im Folgenden werden wir unser ABM einführen und diskutieren. Die Ergebnisse, die später diskutiert werden, basieren auf der statistischen Analyse von 200 Simulationen. Der konkrete Ablauf einer Simulation ist in Tabelle 1 zusammengefasst.

Im Modell gibt es drei Typen von Agenten: Anbieter, Kunden und Netzwerkbetreiber. Die Anbieter bieten ein Produkt an, das von den Kunden nachgefragt wird. Eine Geschäftsbeziehung zwischen Kunde und Anbieter kann nur zustande kommen, wenn beide zum Zeitpunkt des Geschäftsabschlusses beim gleichen Netzwerkanbieter registriert sind. Der Netzwerkanbie-

\begin{tabular}{|c|c|}
\hline $\begin{array}{l}\text { Zeit- } \\
\text { punkt }\end{array}$ & Vorgehen \\
\hline 1 & Agenten werden erstellt. \\
\hline 2 & $\begin{array}{l}\text { Die Kunden und Anbieter werden alle zufällig einem Netzwerk- } \\
\text { betreiber zugeordnet. }\end{array}$ \\
\hline 3 & $\begin{array}{l}\text { Die Simulation läuft für } 500 \text { Zeitschritte. Jeder Zeitschritt besteht } \\
\text { aus } 6 \text { Abschnitten. }\end{array}$ \\
\hline 3.1 & Die Transaktionen werden durchgeführt. \\
\hline 3.2 & Anbieter/Kunden zahlen Gebühren an Netzwerkbetreiber. \\
\hline 3.3 & Die Netzwerkbetreiber zahlen Fixkosten und Nutzerkosten. \\
\hline 3.4 & Die Daten für den Zeitschritt werden gesammelt. \\
\hline 3.5 & $\begin{array}{l}\text { Die Anbieter/Kunden entscheiden, einem neuen Netzwerk bei- } \\
\text { zutreten und ein bestehendes zu verlassen. Die Netzwerkeffekte } \\
\text { werden entsprechend aktualisiert. Sie treten einem zufälligen } \\
\text { neuen Netzwerk bei, wenn sie nach Abzug aller Kosten noch mehr } \\
\text { Geld als die Registrierungsgrenze zur Verfügung haben. }\end{array}$ \\
\hline 3.6 & $\begin{array}{l}\text { Die Netzwerkbetreiber updaten ihre Strategie und setzen Preise } \\
\text { gemäß ihrer aktuellen Strategie. }\end{array}$ \\
\hline
\end{tabular}

Tab.1: Ablauf einer Simulation. Für eine detailliertere Beschreibung siehe Heinrich und Gräbner (2017). 
ter bietet den Kunden und Anbietern sein Netzwerk gegen ein Entgelt zur Nutzung an.

Das Verhalten des Netzwerkbetreibers ist entscheidend für die Dynamiken auf zweiseitigen Märkten und steht im Fokus des Modells. Entsprechend werden die Anbieter und Kunden möglichst simpel modelliert: Zu Beginn der Simulation startet jeder Anbieter und jeder Kunde mit einem Vermögen von 0. Durch jede erfolgreiche Transaktion erfahren jeder Anbieter und jeder Kunde einen Nutzen. Dieser potenzielle Transaktionsnutzen $b^{i}$ wird für jede Transaktion zufällig aus einer Gleichverteilung neu bestimmt.

In jedem Zeitschritt eines Simulationsdurchlaufes kann es zu einer parametrisch bestimmten Maximalzahl an Transaktionen kommen. Für eine Transaktion werden ein Kunde und ein Anbieter zufällig ausgewählt. Die Transaktion wird durchgeführt, wenn beide bei dem gleichen Netzwerkbetreiber registriert sind und beiden nach Abzug aller Kosten ein positiver Nutzen der Transaktion bleibt. Jeder Kunde und Anbieter hat einmal pro Zeitschritt die Möglichkeit, seine Registrierung bei einem Netzwerkanbieter zu kündigen bzw. sich bei einem neuen Netzwerkanbieter zu registrieren. Die Agenten werden eine Registrierung kündigen, falls die Kosten der Registrierung in der letzten Periode den Nutzen überstiegen haben. Für die Anbieter/ Kunden ist es attraktiver, in ein Netzwerk einzutreten, in dem viele Agenten der anderen Kategorie bereits Mitglied sind, da dies die Wahrscheinlichkeit für eine positive Transaktion erhöht. Dies führt zu den für zweiseitige Märkte charakteristischen Netzwerkeffekten.

Der Netzwerkbetreiber muss über die Höhe der Gebühren entscheiden, die er (i) für die Mitgliedschaft in seinem Netzwerk von Anbietern und Kunden erhebt und (ii) von Anbietern und Kunden pro Transaktion erhebt. Da sich die Preise für Anbieter und Kunden unterscheiden können, muss er vier Preise setzen.
In unserem Modell, das in Heinrich und Gräbner (2017) ausführlich und quantitativ analysiert wird, können wir die Effekte verschiedener Preissetzungsstrategien studieren. Es ist auch möglich, rationale Optimierung mit perfekter Information, wie sie in den herkömmlichen Modellen zweiseitiger Märkte verwendet wird, zu implementieren. Ein solches Design verlangt jedoch sehr unrealistische Annahmen, z. B. dass der Netzwerkanbieter die Nachfragekurven der Anbieter und Kunden kennt. Selbst unter diesen Bedingungen ist es ihm jedoch nicht immer möglich, erfolgreich Preise zu setzen (Heinrich und Gräbner 2017). Hier konzentrieren wir uns daher auf folgende Fälle:

1. Ein oder mehrere Netzwerkanbieter setzen die Preise auf Basis ihrer Erfahrungen in der Vergangenheit.

2. Ein oder mehrere Netzwerkanbieter setzen die Preise wie in (1.), sie verändern die Preise aber nicht mehr, sobald sie ein angemessenes Gewinnniveau erreicht haben.

Den in beiden Fällen verwendeten Entscheidungsmechanismus der Provider heißt reinforcement learning: Jeden der vier Preise kann ein Netzwerkbetreiber erhöhen, senken oder unverändert lassen. Wenn er durch eine Strategie seinen Gewinn steigern konnte, wird er diese Strategie in Zukunft häufiger verwenden. Wenn sein Gewinn dagegen verringert wurde, wird er die entsprechende Strategie in Zukunft seltener verwenden. Diese sehr einfache Heuristik spiegelt gut das Prinzip von trial and error wider. Zahlreiche Untersuchungen haben die Verwendung und den Erfolg solch simpler Heuristiken gezeigt (Bingham und Eisenhardt 2011). Die recht einfache Reinforcement-learning-Heuristik scheint uns für eine realistische Abbildung von Preissetzungsstrategien zu simpel, sie ist aber ein guter Ausgangspunkt für unsere Analyse und immer noch deutlich realistischer als die Annahme eines rationalen Netzwerkbetreibers, der in der Praxis oft zu gar keiner Entscheidung kommen kann.

Parameter der Simulation

\begin{tabular}{|c|c|c|}
\hline Anzahl der Kunden & - & 10.000 \\
\hline Anzahl der Anbieter & - & 2.000 \\
\hline Anzahl der Netzwerkbetreiber & - & 1 oder 10 \\
\hline Fixkosten für Netzwerkbetrieb & - & 10.000 \\
\hline Fixkosten pro Netzwerkkunde & Die Kosten, die dem Netzwerkbetreiber für jeden registrierten Kunden/Anbieter entstehen & 50 \\
\hline Variable Kosten für Transaktionen & Die Kosten, die dem Netzwerkbetreiber für jede durchgeführte Transaktion entstehen & 25 \\
\hline Zeitschritte & - & 500 \\
\hline Mögliche Transaktionen pro Zeitschritt & - & 30.000 \\
\hline Registrierungsgrenze & $\begin{array}{l}\text { Wenn der Gewinn von Kunden/Anbietern unter dieser Grenze liegt, registrieren sie sich } \\
\text { auf keinen Fall bei einem weiteren Netzwerk }\end{array}$ & 400 \\
\hline Wertebereich für Registrierungspreise & - & $(-3.000,5.000)$ \\
\hline Wertebereich für Transaktionspreise & - & $(-1.000,1.000)$ \\
\hline
\end{tabular}




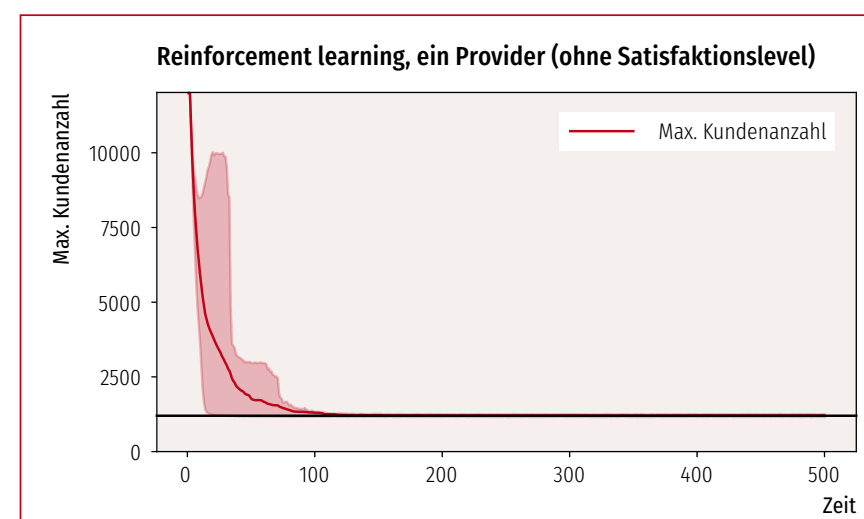

Reinforcement learning, zehn Provider (ohne Satisfaktionslevel)

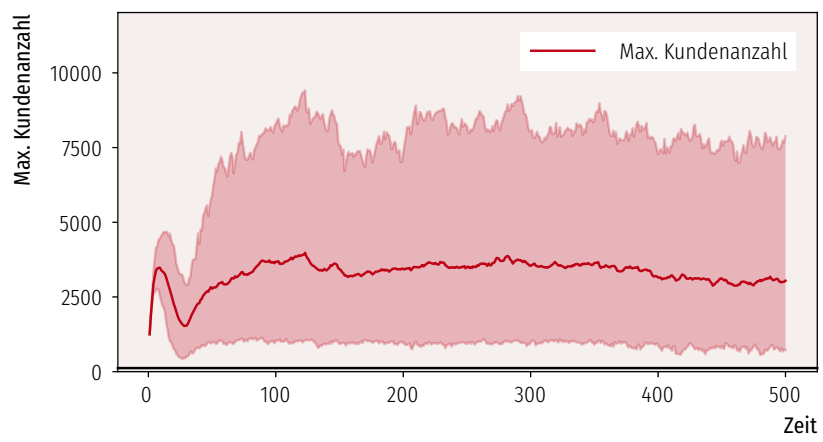

Reinforcement learning, ein Provider (mit Satisfaktionslevel)

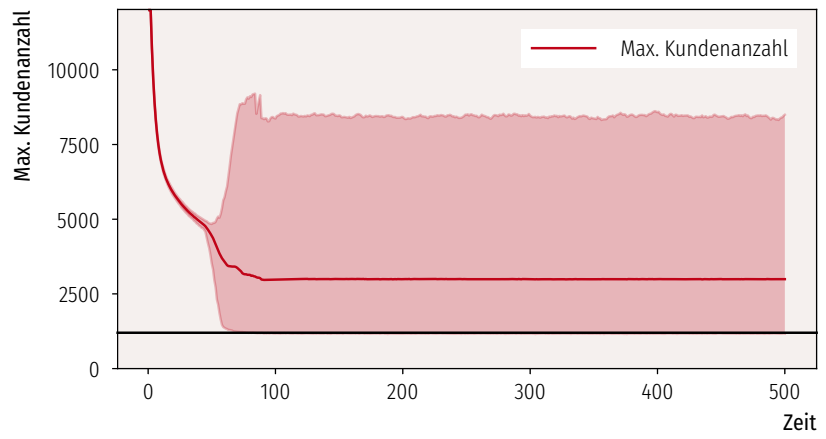

Reinforcement learning, zehn Provider (mit Satisfaktionslevel)

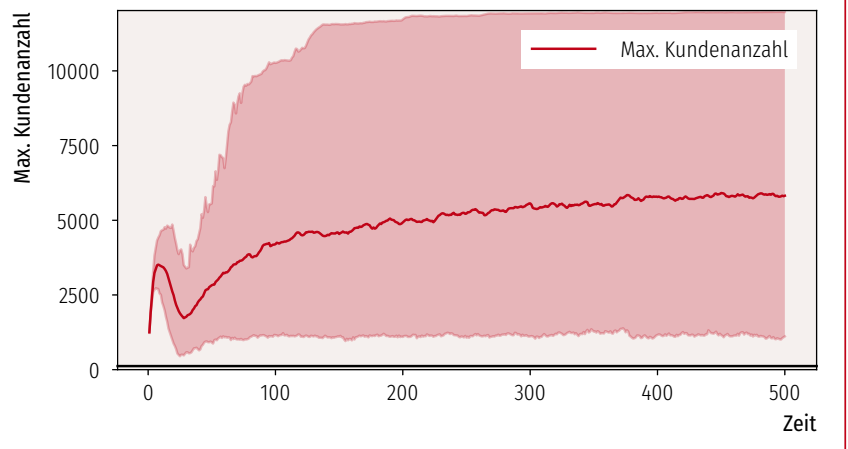

Abb.1: Ergebnisse der Simulation für vier verschiedene Settings: jeweils ein oder zehn Netzwerkanbieter mit oder ohne Satisfaktionslevel. Die rote Linie gibt den Mittelwert von 200 Simulationsdurchläufen an, die $90 \%$ Quantile sind farblich markiert.

Quelle: Heinrich und Gräbner (2017, S. 15)

Die Parameter unseres Modells sind in Tabelle 2 zusammengefasst. Für eine Sensitivitätsanalyse siehe Heinrich und Gräbner (2017). Wir werden nun untersuchen, inwiefern die Netzwerkanbieter mit simplen Heuristiken in der Lage sind, ein für sie lukratives Netzwerk aufzubauen, und welche Implikationen dies für die Effizienz des Gesamtsystems hat.

\section{Ergebnisse und Diskussion}

Die Simulationen zeigen, dass unsere Modellökonomie nicht zu einem eindeutigen Gleichgewicht konvergiert, sondern eine pfadabhängige Entwicklung vollzieht. Die Konkurrenz zwischen verschiedenen Netzwerkanbietern ist eine wichtige Voraussetzung für die Stabilität und Funktion zweiseitiger Märkte. Wie in Abbildung $1 \mathrm{zu}$ sehen ist, brechen die Netzwerke eines einzelnen nicht satisfizierenden Netzwerkanbieters relativ zügig zusammen. ${ }^{2}$ Dass die Zahl der registrierten Nutzer nicht unter $10 \%$ der Gesamtzahl fällt, ist ein Artefakt unseres Simulationsdesigns, das für das grundsätzliche Verständnis jedoch zweitrangig ist. Ein einzelner satisfizierender Netzwerkanbieter schafft

2 Dass die Zahl der registrierten Nutzer nicht unter $10 \%$ der Gesamtzahl fällt, ist ein Artefakt unseres Simulationsdesigns. Siehe Heinrich und Gräbner (2017) für Details, die für das grundsätzliche Verständnis jedoch zweitrangig sind. es häufiger, ein funktionierendes Netzwerk aufzubauen, jedoch sind die Ergebnisse schlechter als für den Fall mit zehn Netzwerkanbietern.

Der Grund liegt in dem Selektionsmechanismus, der durch die Existenz verschiedener Netzwerkanbieter ausgelöst wird: Anbieter und Kunden werden regelmäßig zu den Netzwerkanbietern mit der für sie kosteneffizientesten Preisgestaltung wechseln. Netzwerkanbieter mit unattraktiven Preisangeboten werden aus dem Markt gedrängt. Dies funktioniert nur, wenn genügend Netzwerkanbieter am Markt sind. Erstaunlicherweise ist das Ergebnis eines Wettbewerbs jedoch nicht nur für die Anbieter und Kunden von Vorteil, sondern auch für die Provider selbst: Ohne die Konkurrenz anderer verrennen sie sich in nachteiligen Preissetzungsstrukturen, und das gesamte System bricht oft zusammen. Im Konkreten ist die Heuristik nicht in der Lage, den Zusammenbruch aufzuhalten, sobald die ersten Nutzer das Netzwerk verlassen und damit den Netzwerknutzen für andere reduzieren. In realen zweiseitigen Märkten existiert dieser Effekt in der Regel nicht, da dort kein vollständig konnektives Nutzernetzwerk (jeder Verkäufer mit jedem Käufer verbunden) zugrunde liegt. Lokale Einbrüche haben nicht notwendigerweise direkte Auswirkungen auf andere Teile, dies gibt der Plattform Korrekturspielraum. Unhaltbare Preissetzungsstrukturen, die das Wachstum des Netzwerkes lokal stören, sind in realen Systemen mit Netzwerkexternalitäten dennoch zu erwarten und auch zu beobachten. 


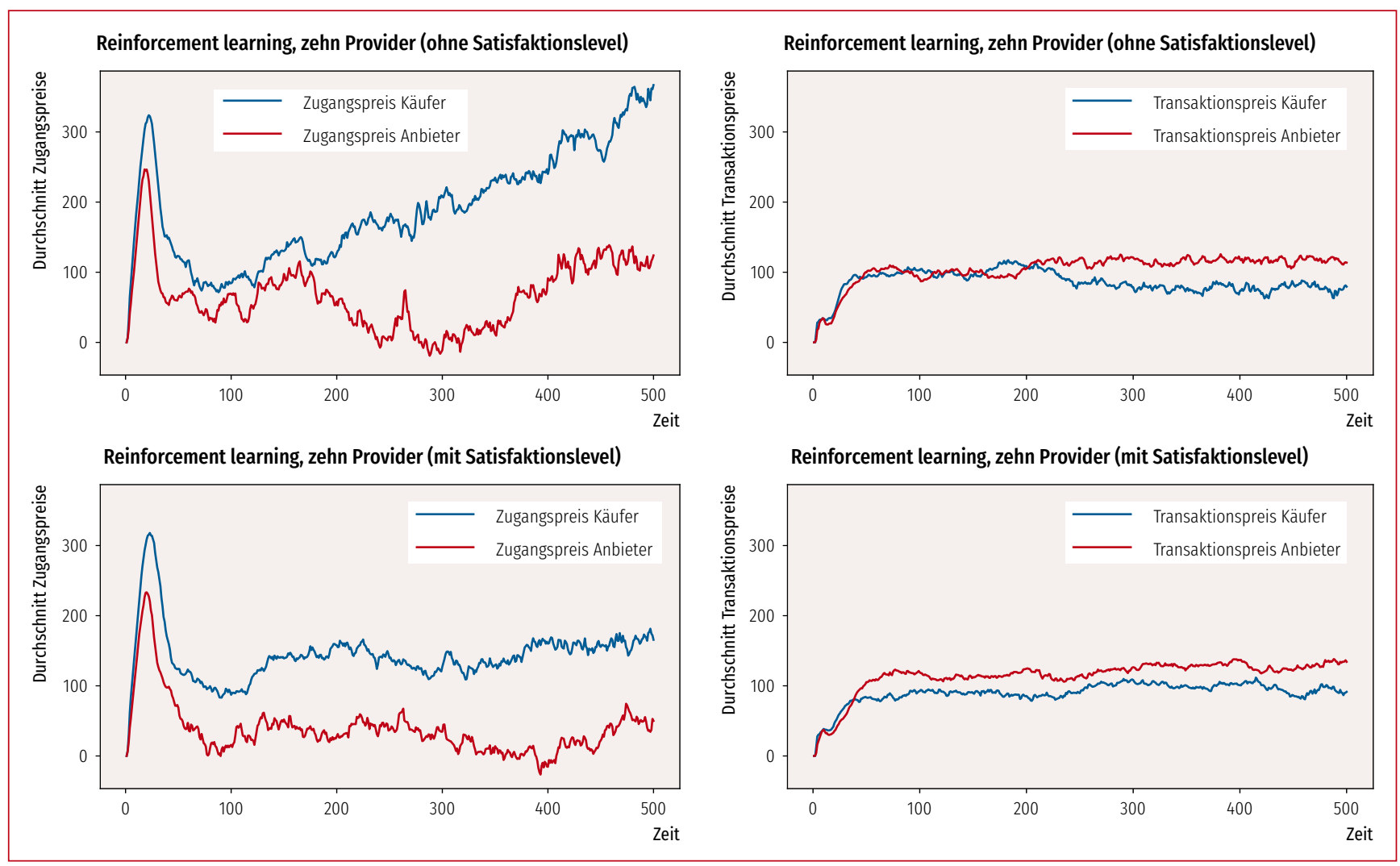

Abb.2: Die Zutritts- und Transaktionspreise, die im Falle von 10 Netzwerkanbietern vom erfolgreichsten Netzwerkbetreiber gesetzt werden. Während die kleinere Gruppe geringere Registrierungsgebühren zahlen muss, sind ihre Transaktionspreise höher als die der größeren Gruppe.

Die Darstellungen in Abbildung 1 führen direkt zum zweiten Ergebnis unserer Simulation: Satisfizierende Netzwerkbetreiber sind erfolgreicher als nicht-satisfizierende. Der Versuch ständigen Optimierens dagegen führt oft zu überzogenen Preisforderungen oder zu günstigen Preisen.

Interessanterweise entwickeln die Netzwerkbetreiber jedoch selbst mit einer simplen reinforcement learning Heuristik ausgeklügelte Preismechanismen. Wie in Abbildung $2 \mathrm{zu}$ sehen, verlangen die Netzwerkanbieter von der zahlenmäßig größeren Gruppe (in unserem Falle die Kunden) deutlich höhere Zugangspreise. Mit diesen höheren Zugangspreisen können die niedrigen Zugangspreise für die kleinere Gruppe finanziert werden. Letztere ist für den Betreiber wichtig, damit genügend Transaktionen stattfinden können und der Anreiz für die größere Gruppe steigt, Mitglied im Netzwerk zu werden. Auf der anderen Seite ist es sinnvoll, die zahlenmäßig kleinere Gruppe mit höheren Transaktionspreisen zu belegen, da hier die erwartete Anzahl von Transaktionen höher liegt.

Herkömmliche Gleichgewichtsmodelle lassen eine solche Differenzierung der Preise in der Regel nicht zu. Es sind jedoch genau diese Dynamiken, die aktuell im US-amerikanischen Kreditkartenmarkt zu beobachten sind: Während Anbieter teils hohe Gebühren für den Service zahlen müssen, werden die Kunden durch attraktive Prämien zum Erwerb einer Kreditkarte animiert.

\section{Zusammenfassung und Ausblick}

Wir haben argumentiert, dass zweiseitige Märkte ein essenzieller Bestandteil moderner Volkswirtschaften sind. Zudem haben wir ein einfaches agentenbasiertes Modell (Heinrich und Gräbner 2017) eingeführt und dessen Ergebnisse diskutiert. In Fallbeispielen lässt sich eine Vielfalt verschiedener Ausformungen zweiseitiger Märkte identifizieren. Entscheidend ist, dass in der Regel nur die (zumindest temporär) erfolgreichen Fälle untersucht werden können, da für andere keine oder nur wenige Daten verfügbar sind. Während die beobachtete Vielfalt auf pfadabhängige Prozesse hindeutet, wie sie auch im ABM beobachtet wurden, legen die vorliegenden Beispiele nahe, dass der strategische Fokus der Plattform eher auf Stabilität und Vergrößerung der Nutzerbasis als auf Ausbeutung des bestehenden Netzwerkes liegt.

Im Modell erreichen erfolgreiche Netzwerkbetreiber - verglichen mit anderen Marktformen - eine große polit-ökonomische Machtposition. Auf Produkte kann man verzichten, nicht-vernetzte Anwendungssoftware kann man aufgeben, Elektrizität kann man von einem anderen Lieferanten beziehen. Aber der Wechsel der Plattform (etwa Facebook, Microsoft Windows/Office oder in einem Online-Marketingzusammenhang Google) kann unmittelbare Folgen für die Handelsbeziehungen oder das soziale Umfeld haben. Die Daten, die der Plattform vorliegen, 
geben darüber hinaus ein Bild der sozialen und ökonomischen Mikrostruktur der Gesellschaft. Wohin führt uns diese einseitige Machtposition? Man denke an die Berichte über die Bedeutung geschickter Datenanalyse und politischer Fake-News-Portale für den Ausgang demokratischer Wahlen. Auch wenn diesbezügliche Berichte zum gegenwärtigen Zeitpunkt noch übertrieben erscheinen, sind technologie- und wirtschaftspolitische Erwägungen zur Zukunft der Lenkung, Förderung, und Regulierung zweiseitiger Märkte dringend geboten.

Denkbare Politikmaßnahmen sind beispielsweise die Unterstützung entstehender Systeme zur Förderung von Entwicklung und Vielfalt, gesetzliche Vorschriften zur Garantie von Kompatibilität, sowie gesetzliche Beschränkungen von Preiserhöhungen, insbesondere bei monopolähnlichen Marktsituationen. Möglich, aber kostenintensiv, wäre auch die staatliche Bereitstellung der Infrastruktur. In diesem Fall wäre der Netzwerkbetreiber ein öffentliches Unternehmen. Ein Beispiel hierfür wäre der Aufbau des Internets durch das US Department of Defense, auch wenn das Internet nicht als die zweiseitige Plattform vorgesehen war, die es heute ist (mit Website- und Serverbetreiber*innen auf der einen und Websitebesucher*innen auf der anderen Seite). Erweiterungen unseres Modells können dazu beitragen, Kosten und den zu erwartenden Erfolg dieser und anderer Politikmaßnahmen zu eruieren.

\section{Literatur}

Bingham, Christopher; Eisenhardt, Kathleen (2011): Rational Heuristics: The

„Simple Rules“ that Strategists Learn from Process Experience. In: Strategic

Management Journal 32 (13), S. 1437-1464

Boerner, Lars; Quint, Daniel (2010): Medieval Matching Markets. In: School of

Business \& Economics. Discussion Paper. Berlin: Freie Universität Berlin. DOI:

10.2139/ssrn.1727700.

Eisenmann, Thomas; Parker, Geoffrey; van Alstyne, Marshall (2011): Platform

Envelopment. In: Strategic Management Journal 32 (12), S. 1270-1285.
Heinrich, Torsten; Gräbner, Claudius (2017): Beyond Equilibrium: Revisiting Two-Sided Markets from an Agent-Based Modelling Perspective. In: International Journal of Computational Economics and Econometrics. Im Druck. Hoffmann, Birte; Roos, Michael (2012): Agentenbasierte Modelle in der Makroökonomik. Potenziale und Probleme. In: Martin Held, Gisela Kubon-Gilke und Richard Sturn (Hg.): Jahrbuch Normative und Institutionelle Grundfragen der Ökonomik. Marburg: Metropolis, S. 213-233.

Lehtinen, Aki; Kuorikoski, Jaako (2007): Computing the Perfect Model: Why Do Economists Shun Simulation? In: Philosophy of Science 74 (3), S. 304-329.

Meyer, Tobias Georg (2012): Path Dependence in Two-Sided Markets. Promotionsschrift. Berlin: Freie Universität Berlin.

Peitz, Martin; Rady, Sven; Trepper, Piers (2011): Experimentation in Two-Sided Markets. In: CEPR Discussion Paper DP8670. London: Centre for Economic Policy Research.

Rochet, Jean-Charles; Tirole, Jean (2006): Two-Sided Markets: A Progress Report. In: The RAND Journal of Economics 37 (3), S. 645-667.

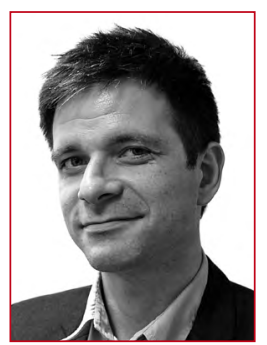

\section{DR. TORSTEN HEINRICH}

arbeitet und lehrt in den Bereichen Komplexitätsökonomik, evolutorische Ökonomik, agentenbasierte Modellierung und Spieltheorie. Inhaltlich arbeitet er zu Industriestruktur und zu technologischen Aspekten ökonomischer Entwicklung, etwa Innovationssystemen und Open Source Software.

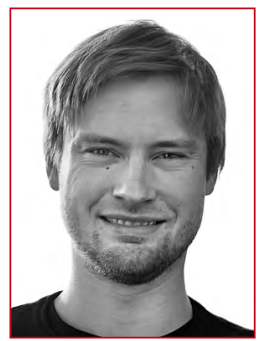

\section{DR. CLAUDIUS GRÄBNER}

forscht methodisch im Bereich der Komplexitätsökonomik und agentenbasierter Modellierung und untersucht weiterhin die Struktur internationaler Handelsnetzwerke, die sozioökonomischen Effekte volkswirtschaftlicher Offenheit und die innovationsökonomischen Grundlagen wirtschaftlicher Entwicklung.

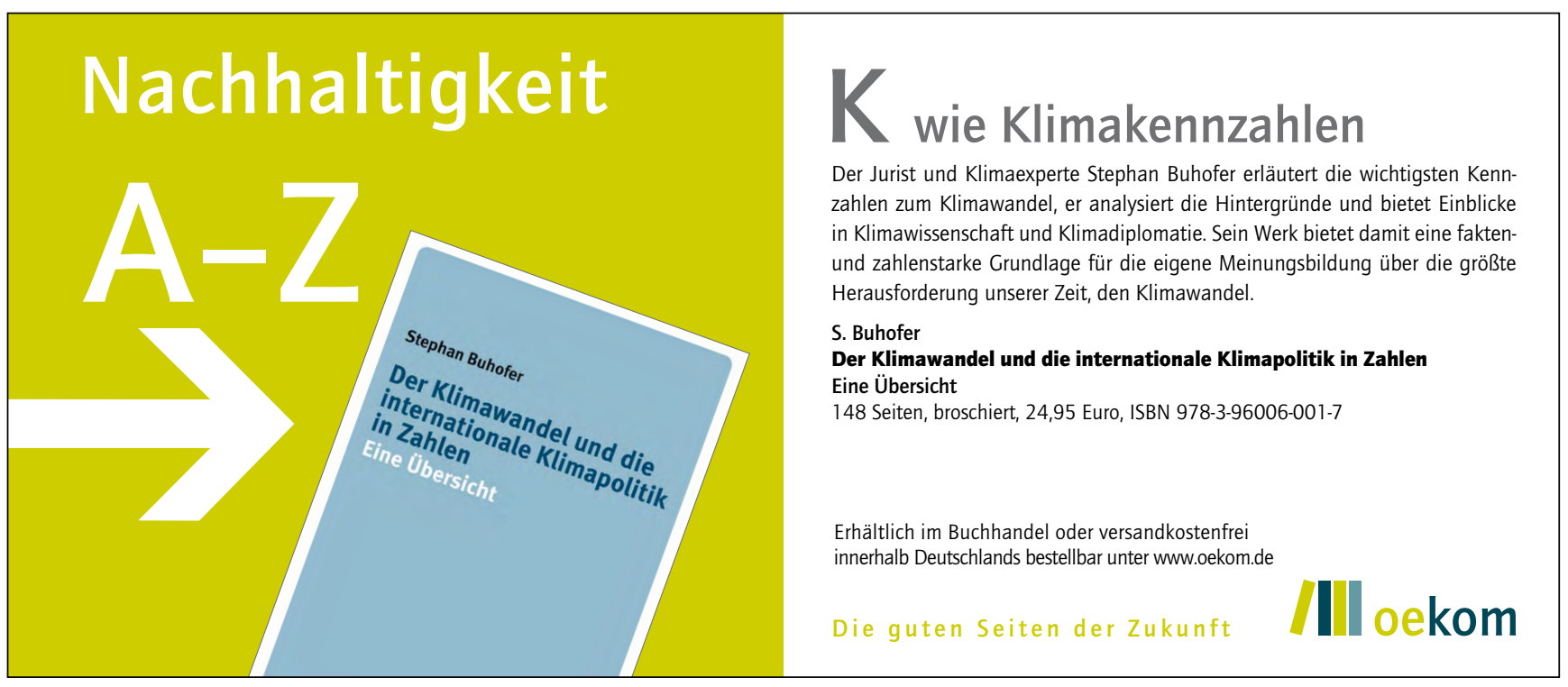

